\title{
The Skype paradox
}

\section{Homelessness and selective intimacy in the use of communications technology}

\author{
Richard Harper, Rod Watson and Jill Palzkill Woelfer \\ Social Shaping Research, Cambridge / Telecom ParisTech, Paris / \\ University of Washington, United States
}

Digital technologies are likely to be appropriated by the homeless just as they are by other segments of society. However, these appropriations will reflect the particularities of their circumstances. What are these appropriations? Are they beneficial or effective? Can Skype, as a case in point, assuage the social disconnection that must be, for many, the experience of being homeless? This paper analyses some evidence about these questions and, in particular, the ways communications media are selected, oriented to and accounted for by the homeless young. Using data from a small corpus of interviews, it examines the specific ways in which choice of communication (face-to-face, social media, or video, etc.), are described by these individuals as elected for tactical and strategic reasons having to do with managing their family relations. These relations are massively important both in terms of how communications media are deployed, and in terms of being one of the sources of the homeless state the young find themselves in. The paper examines some of the methodical ways these issues are articulated and the type of 'causal facticity' thereby constituted in interview talk. The paper also remarks on the paradoxical problem that technologies like Skype provide: at once allowing people in the general to communicate but in ways that the homeless young want to resist in the particular. The consequences of this for the shaping of communications technology in the future are remarked upon.

Keywords: sociology, ethnomethodology, philosophy of action, homelessness, family, communication media, Skype, membership categorization, participation frameworks, reasons 


\section{Introduction}

'Homelessness' is a polysemic term, both in everyday parlance and in professional usage. It can be either a category on its own behalf, 'the homeless', or a predicate and modifier of other categories, such as the 'homeless young person', and so forth. Its particular meaning is bound, also, to the cultural and historical moment in which it is used and the related categories thus implied. An early study of homelessness is Nels Anderson's (1923) classic book, The Hobo, for example: here homelessness is tied to the auditory rumble of trains entering and leaving Chicago, the murmur of strangers in a boarding house, the ad hoc economics of casual farm work, and a credo that led to its own vernacular, self-identifying label for those in this world, the "hobo". Today, in contrast, homelessness in common sense terms evokes and is tied to other matters - the bleakness of life on the urban street; to how incomes, if any are to be found, derive from agencies and not seasonal work, and a world connected not by railways and cars but by Facebook, Skype and the smartphone. Moreover, professional explanations offered for homelessness have also changed over time: when Anderson wrote, these were often cast in terms of social cohesion (or fragmentation) bound in part to economics, today such explanations are organized around the topic of social exclusion with all its moral overtones. This in turn is linked to the theoretical motifs of the current time; today these include whether the homeless are also 'mobile' (Jackson 2012, 725-41.) Techniques for examining the use of categories like homelessness need, then, to consider the conceptual worlds of which such a category are a part, worlds which are framed on the one hand by conceptual apparatus of theory and on the other by the material circumstances of experience and self-definition.

Anderson himself had been a hobo, and studied the social and cultural world of such individuals 'from within' - his book is an analysis of the work they sought, the places they inhabited, the terms they used. In his view, this world had to be contrasted with views 'from the outside', those constructed by political commentators, say, a Chicago inspired 'ethnographer' (such as Anderson himself), or from the views of the theoretically driven sociologist of 'mobility' such as Jackson. Even though the world that Anderson explores has now long gone in that form, we consider that the approach we take in this paper echoes in part a primary concern of Anderson himself. Just as he was interested in 'taking the role of the acting subject' rather as a participant observer might do, so we are interested in how ordinary people themselves specify and use the description 'homeless' as part of the vocabulary that articulates their own circumstances, and how they cast their situation and how they cope with that situation, in terms of that specification. We are interested in how this use constitutes a work of a particular order - the work of 
'participants' or 'members', as it were, the work (broadly speaking) of 'doing being homeless' when homelessness is an oriented-to matter at hand. This is not only to do with how a person might deploy a self-description as 'homeless', however; this work is done in a particular context, in a world - today's world and not that of the hobo - whose features make that categorisation problematic in various ways: tied in complex and subtle linguistic webs to other categories and implied actions, as we have noted above. As a case in point, if today's world is often characterised as digitally connected, then being homeless may only allude to issues of place. If Anderson's hobos were cast asunder from their domestic networks, their parents, their siblings, by being in a different world than these individuals, so today, when communication systems seem ubiquitous, then the functionality of the category 'homeless' might imply questions about whether having that status means being geographically separated from home or rather, only being out of touch with all those who made home where ever it might be. For if we are to believe Castells (1996, 1997, 1998) and other sociologists of the digital, this could be seen as a greater price to pay than facts of geography. Consequently, for the term 'homeless' to have salience, for it to evoke, say, a concern that is a matter of 'legitimate complaint' (Schegloff 2005), then those who use that term might have to navigate their way to explaining 'just what' the problem is that this use implies: does it mean 'only' having 'no fixed accommodation', for instance, or does it imply, as well, questions of contact, of being out of touch when being in touch is viewed as so consequential? 'One might be homeless, but one can always Skype' is a phrase that comes to mind.

Leaving aside the practical problem realities of such choices, like who will pay for such a call, what is sure is that applying the term homeless means 'aptly fitting' it into other related or implied categories. Here is labour of a kind, linguistic work of deploying the term in ways that connect this category to others in a web of elaborated meaning. 'Communication', 'being in touch' are likely to be closely related to homelessness, as we say, but doubtless others will also be important. The world is not encapsulated in a single term, but through the delicate, complex appropriation of categories irredeemably dependent upon the elaboration of other categories, words about the world. Getting this use right, or at least getting this use to result in the right actions thereafter, is the pragmatic outcome of utterances: this is why the labour is worth doing. This is the 'pay-off' that derives. Saying that one is homeless has little value unless it comes to be acted upon in a practical way, in practical circumstances. And this, in the first place, requires that this status is commonly agreed, intersubjectively constituted as a fact known in common; one that the respective world endorses, at least in the sense of a working agreement. Although we might 'only' be talking about words, this work, as we are calling it, turns into a move in the politics of living. 


\subsection{Methods and approach}

The concerns of the paper are then essentially in pragmatics, though focused less on the elaboration of the systematic meaning of homelessness than in the practical use of the term in negotiated orders of interaction, in talk, especially, which it treats as the crucible of social life. The paper thus has its roots in ethnomethodological perspectives (Garfinkel 1967, Garfinkel \& Sacks, 1970) and conversation(al) analysis (Sacks, 1992). The concept of membership categorization devises and their analysis, originally expounded by Sacks and developed by Maynard (1988) and more recently by Fitzgerald and Housley (2015) and Watson (2015) is especially pertinent to our approach. For us, this view is a natural development from starting with Anderson's initial methodological stance: a concern for the subject and their point of view, their reasoning. In our case, it's not just their point of view that matters, but how those who have this point of view act as and when they describe that view, and how they deal with what that articulation implies once thrown into dialogue with others - such as ourselves, when we undertake interviews with them.

The move to a concern for language use has important implications for the generalisations and insights that can be generated from research and these are worth bearing in mind from the outset. If at the time that Anderson was writing (and for many years thereafter), the general tenor of sociological inquiry has been to offer generalisations about the homeless that seek, as it were, to correct everyday, lay understandings, so the activities we propose to investigate are about how such spoken understandings, articulations about one's predicament, are built into the actions of the homeless and those whose daily business entail that. In this respect, our analysis intersects with that of Jackson (2012) though her concern is focused on the business of movement as a matter for the homeless young; ours is in the business of description and accounts; broadly speaking, what one might say is the pragmatics of meaning.

In our view, this meaning comes out of, and is constituted by, the methodical ways the world is talked about in self-referring descriptions, in talk about homelessness by the homeless themselves. The talk we examine and attempt to explicate might be conceived as producing a world -in -common and points toward how talk itself, in its features and in its finessed production, constitutes the skilled deployment of know-how about that world and how it might be described. Fundamental to this know-how is recognising that talk is not 'just' verbal description, but descriptions (amongst other things) organised so as to achieve practical ends. Thus, part of this know-how entails using what one might say are laic technique(s) to attain those ends - the practical skills of the ordinary, including, in this case the homeless. Hence when someone self-describes themselves as homeless, though they may have few material possessions, their skill in this self-descriptive work, their adroit use of 
technique and know-how about the world shown in and through their talk, makes them have things in common with those who have much more material wealth, indeed even 'addresses' and 'zip codes' they can call 'their own', and who may well not, by dint of that, have the same worlds in mind when they talk. Both the materially rich and the materially poor, those with addresses and those without, have the material skills of talk - embodied ways of telling the world and thereby making it. Talk, in other words, its skillful use, is the common instrument of this know-how whatever purpose is at hand, as Maynard notes (1988, 311-334).

\subsection{The relation between this approach and other ways of addressing homelessness}

If we are looking at the methodical use of the term 'homeless' as part of this skillful work, then this investigation can clearly supplement other views and concerns that elaborate different aspects of the homeless predicament - talk may be central to the pragmatic production of social circumstances but is not the only aspect of those circumstances worth investigation. However, fitting the analysis of talk and more generally, other aspects of language category work needs to be done carefully.

An elaboration of the contrast between what one might grossly describe as analysis of language techniques and 'explanation of social causes' somehow dependent on those techniques, might be helpful here. Doing so can highlight how the approach we want to take allows us to see just how consequential different ways of formulating the world can be for those involved. Consider, for instance, one of the most significant early studies of homeless men on 'skid row', (Rose 1960, see also Spradley 1970). Rose notes that 'middle class' professionals (his label), such as social workers or medical personnel, characteristically refer to skid row inhabitants as 'alcoholics'. Yet their description is problematic for a variety of reasons (idem, 17-19). The main issue is that 'alcoholic'/'alcoholism' are not terms employed by the men on skid row themselves to describe their own identities, lives or practices. Nor does it contain the same cargo as it does for those looking from the outside, Rose's middle classes. Though men on skid row - and it is mostly men - might describe themselves as 'drunks', they frequently modulate that description to be more apposite, describing themselves as, say, occasional drunks, or, at other times, drunks who can hold their liquor, and so on. In these refinements, they don't thereby classify themselves as 'alcoholics'. In their view, that is a different kind of label, with different implications and orders of corrective that follow on from its use. To put it crudely, being alcoholic is a solvable disease, permanent, uncontrollable except through medicine; ending up drinking on skid row a state of mind- temporary, a failure of moral concentration. Responsibility rather than medicine can sort it out. Rose 
wants to argue that how those on skid row categorise themselves is an important component of their motivational schema, and helps explain the scale and scope of their malaise (see also Bourgois 1998a, 1998b). If they view their drinking as a moral culpability, then they view medical perspectives as diminishing their status as people, as competent enough to judge when they have drunk too much and when they need to amend their ways or even 'turn to medicine'. The concern for medical accounts takes the actor out of the act and replaces it with physiological propensities; no wonder the homeless resent it.

A focus on language use does imply, then, not merely need for care and attention concerning words. 'Alcoholism' seems not so distant from the term 'occasional drunk' but as we see it is more consequential than merely a tidier-sounding label. Similarly here with the term homeless - its meaning is as much a question of who uses it as what it might label in terms of location. While studies of language praxis draw attention to the social actors as being skilled and competent, as sophisticated agents in the production of social order, able to demarcate what they are responsible for and what are 'other's concern', they also beg the question of who's work this is. This is our starting-point (Lynch 1993).

The relationship between this and other perspectives on language use is thus consequential and enormously important and reflects this fundamental issue: who speaks. Though for methodological purposes the production of meaning by the subjects studied might be the topic, this does not mean that this is the only way of examining the social problem - other voices matter too. But it does mean that care is required when seeking to fit different explanatory systems together, different ways of talk, especially if these turn around the use of everyday 'laic' concepts - when one form of talk, the everyday, is also used by professional users. Such problems of fitting are of course well recognised in contemporary social theory, with Latour (2013), for example, giving much attention to the problem of language across and between the disciplines as well as between specialist and everyday users. Language makes the world but it can also bewitch.

\subsection{Some remarks on data}

The illustrations or vignettes we bring to bear in this paper derive from a corpus generated by an ongoing series of research activities on homeless young and street life in the USA and England. Prior studies in this series have looked at the problems of how the young maintain digital possessions when 'on the street' (Woelfer \& Hendry 2011) while another has looked at how they articulate their identity through, for example, digital music collections (Woelfer \& Hendry 2010). These research endeavours make it clear that being 'homeless' and young in the cities 
of Seattle, in the US, and Cambridge, in the UK, do not equate to being cut off from the digital. Though these two cities might be quite dissimilar in other respects - scale, income, educational levels and so forth - on this measure they are remarkably alike. The homeless young have extensive, if not continuous access to digital devices, to the Internet and hence to social media, as well as to messaging formats provided by web-based services - email, video telephony (Skype, for example) and similar.

One of the interesting things that came out of this research is whether this digital connectivity reduces the experienced 'severity' of being homeless. With 'perpetual contact' (Katz and Aakhus 2002) the homeless young may, sometimes, have no roof over their heads but even so can share their predicament with family, friends, mum and dad wherever they might be. If they can find access to the Internet, they can always Skype. Given this, one might wonder also why the young find themselves homeless: it would not be because they are out of touch; something, for instance, to do with the kinds of economic activities that they seek must be at issue and these, presumably, are not related to connection or how it might be supported by the digital. However, in our research, it became clear that being constantly in touch with family and with parents in particular is precisely one of the things that the homeless young want to avoid. Indeed so intense does their aversion to being in constant communication with their parents appear to be that it points towards the possibility that problems of communication with parents may be a cause of the young becoming homeless in the first place. This is why we coin the phrase, the Skype Paradox. Being able to communicate easily seems to highlight the problems of communication. These are not technological but social, to do with those involved. It was possibilities such as these that prompted the investigations reported here.

Though, and as we say, prior research looks at the homeless in Seattle and Cambridge, for the purposes of this paper, interview data is used from homeless young people in Cambridge alone. This is only for reasons of length. What we focus on are the systematic ways these individuals, who, like the many others interviewed and surveyed in the larger research programmes, articulate the situated meaning of homelessness and, as they do so, manage its implications in what we will show are methodic ways that echo our other studies. Being in touch with parents, managing this, avoiding certain forms of contact is central to this patterning. These methods are about articulating a young person's status as homeless with all its implied corollaries (such as those related to being in touch with family members) and how they come to acquire their power in intersubjective understandings, as warrants for explaining 'homelessness' both in the general and in the particular, as something that 'makes sense' and which can be viewed as something with a sensible 'history', 
one which may point towards 'things to do' about it - showing sympathy and understanding, offering routes out of this condition, temporary solutions to the need for a roof and so on. This is the pay on effectively 'doing the talk' of 'being homeless'.

Specifically, the evidence, from which the illustrations are drawn, is assembled from the following process. Ten people (seven men, three women) volunteered to a welfare service agency to meet on a one-to-one basis with one of the authors. These individuals, whom we shall variously call respondents and subjects, took part in three separate activities: the first a form filling exercise, required for institutional purposes, then a semi-structured interview on the topic of respondents' circumstances and communication with different people, and, third a communication design exploration where subjects were asked to "imagine a device that could help a homeless young person to talk to their parents", and to draw images of devices that would help us understand the way they oriented to acts of communication (see Yoo et al. 2013 for an overview of this approach; Jackson 2012 does something parallel to this when she examines, e.g., her subjects' drawn depictions of their lives, etc.). The interviews ranged in length from 16-49 minutes and yielded 298 minutes of audio. Interview recordings were transcribed and evidence from some of these are used here. The drawings and other materials are analysed in companion papers.

\section{Analysis}

\subsection{Preliminary observations}

We want to start our remarks by noting that 'talk-in-interviews' is itself a topic of study, no less so than the more general topic of homelessness. But it is through talk-in-interviews that our focused attention and the evidence we bring to bear are based. Our respondents' accounts were collaboratively built up in utterances and actions with us, in interview. Our data, then, do not simply furnish simple conduits to 'what really happened': instead our view on the 'experience' of homelessness is constituted in and through the interviews as interactional events. Thus, in the following Sample 1, one can see how the forms completed in the first part of the interview process framed subsequent steps and topics in the interviews themselves. Note, in this case, the features of the determination 'homeless': the respondent (P. 5) considers himself 'homeless' in a very specific sense and in a sense that has changed for him. He seeks to explain that he has recently been homeless but that he no longer thinks this is the case. For now, at the time of the interview, he has been allocated a room for which he has been waiting. 


\section{Sample 1}

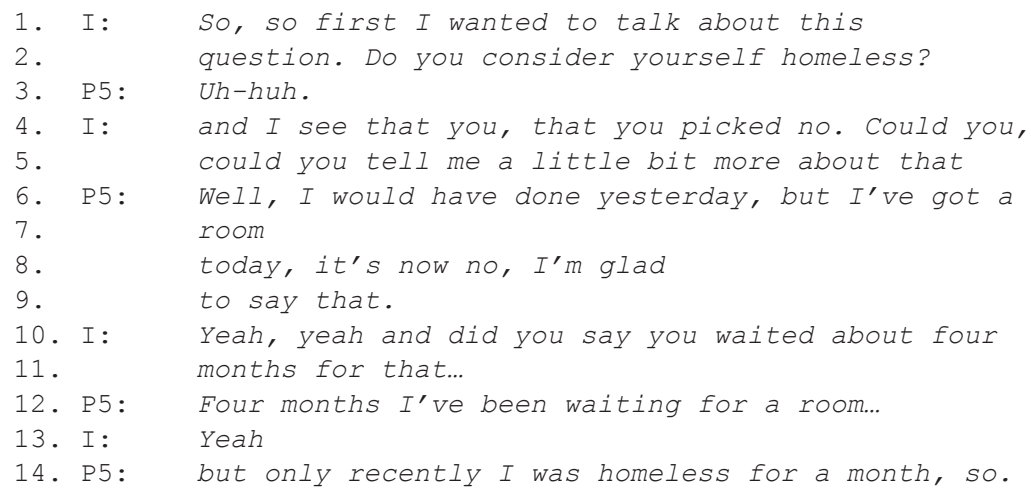

We see here that the determination of 'homeless' connotes not having a room of one's own and this is contrasted with not having a place to stay. In this respect, there is a 'customization' of the concept of homelessness that allows it to fit this person's situation; his personal world. By contrast, six other subjects in our study stated that they were homeless by the very fact of living in a hostel, which some characterized as 'for the homeless'. Some combined this evaluation by alluding to their estrangement from their family of origin and thus from their family home, entangling homelessness, hostel and eviction in one single, overarching status.

The specifics of each biographical case notwithstanding, Sample 1 illustrates how 'homelessness' is a 'range term', covering a number of specific meanings, and used in tailored ways by this respondent (and others) for a particular situation, a particular social world, theirs. Here we see careful formulations used to locate whereabouts a person wants to be seen to be, at a particular point in what Ingold (2011) would say is their dwelling, an achieved weaving of a unique trajectory in a moment in time. In the ways they deploy the category, they are weaving the thread of their own existence into a world known in common: they are giving pointedness to this world by giving it a 'just this' aptness in the interview. As they do so, so they link a particular and nuanced application of one category, in this case the primary category 'homeless', to others that come into play as consequential on the given trajectory of their affairs - hostel, family, and so on.

What we do not see in Sample 1, however, is any relationship between homelessness and matters that might, crudely speaking, be described as 'digital'. If we saw in previous studies how digital technology is used to share content, and through this to create a sense of individuality for those who label themselves as homeless, we saw also that the digital could be used to support 'being in touch'. Being homeless does not strip those with that status from the social fabric of social media - Facebook say; nor does it remove them from web-enabled messaging tools like Skype and Gmail. 
But that said, just who and how one communicates is a matter of great salience to the homeless young; indeed and as we say, an especial concern.

So, and for example, our Cambridge subjects reported to us that, if and when it did occur, communication with, for example, their parents, is conducted on a particular basis, a basis that contains, in some instances, a managed asymmetry in the time-structuring of the interchanges. This, in turn, is located in the spatially-distributed nature of those communications that allows digital mediation of the act of communicating to have special forms - affordances if you like, to do with this asymmetry.

To give a thumbnail sketch or vignette of the kinds of management we have in mind: a young female respondent described to us how she emailed her mother and then closed down her email for several days, so as to occlude the possibility of having to deal with an immediate reply. Managing her email in this fashion wasn't about tempo alone; her account was intended to show how it was also an interactional mode of managing emotions, too. And this meant not only managing her own 'personal' emotions but the emotional scheme pertaining between her and her mother, the participants in the act, however spread over time or mediated by technology, they might be.

What our respondent was offering us in interview was then the claim that messages 'from a mother' that may be 'emotionally charged' or that may elicit an 'immediate emotional response' from herself, might be managed in this way. Her account, which we are offering only as sketch here, sought to posit the notion that asynchronicity of communication, broadly speaking, could be a tool that allows participants to deal with the substance of the communication when that substance was of a particular order; emotional, angry, provocative. We should note, of course, that this is a 'technique' that anyone can use, not just the homeless; it is as it were, commonplace. What we learned in our interviews with this respondent is that she could, or would, deal with 'emotion' by taking her own good time, for example - just as anyone would do. Her account to us is not meant to explain the uniqueness of her acts, but the sense of them. This points to the phenomenon of using techniques that are available to anyone, but using them in highly specific ways that are organized around the situated us of the term 'homelessness'-or homelessness in-this-case.

Another vignette might be helpful here: a young male reported posting a message on Facebook, knowing that his parents would disagree with what he had to say. He explained that if his parents 'over-reacted' then other members of the social network (i.e., Facebook) might, given time, take on a mediating role, 'cooling out' the parents or perhaps 'cooling them down' through their own discreet communications,- their postings, for example, even a direct message through Facebook. Facebook, then, worked for this person as an interactional management device in relation to certain 'sensitive topics' that his parents might consider to be 
'inflammatory' and where, therefore, direct, unmediated communication with them might be 'emotionally-loaded' - just as they were in the prior example, of mother and daughter. But again, the account was offered not to show how the predicament of this homeless male is unique, so much as to also show how efforts to remedy its features involve commonly understood techniques - ones related to how Facebook behaviour is, in a sense, public, and hence subject to constraints to which private face-to-face communication might not be subject. To put it differently: mediating communication via this social networking system involves third parties and this can thereby deliver a 'mitigating' or 'cushioning' effect on the character or 'feel' of the communication in question. And from what the young man explains, this is likely and sought for in the context of what is otherwise a communication dyad, a relational pair - this son and his mother.

A third: another respondent told us that he would never use Skype or other forms of video-communication to chat with his parents since that would mean that he would have to look at them and they at him. Consequently, he would immediately see their reactions to everything he said. He explained to us, in effect, that unmediated by any third party, such synchronous communications are not insulated against say, spontaneous, perhaps unguarded, rage, impatience, frustration or admonishment. What the parents could see or hear in a Skype call could lead to such reactions. Hence this can be why 'Skyping' is to be avoided, in this person's view.

These vignettes point towards time-structuring communication in various ways so as to be 'asynchronous'. We are not making a categorisation in terms of the technology, though the properties of the technology are perceptible resources for this. Nor are we wanting to piggy-back on the use of the term 'synchronous' and 'asynchronous' as it used by engineers of communications technology. We are labeling how this characterisation describes the in vivo management of communicative acts by those we interviewed. And what we see is that communication when one is homeless requires managing - managing to achieve a certain kind of asynchronicity. As should be clear, this is often artful work; what should also be clear is that this work has something to do with the status that young people find themselves in. Though anyone might use Facebook, something about the specifics of those we interviewed made doing so especially important: for it allowed one of a relational pair to be perceptibly 'controlled' by the other; for mum to be 'cooled down' so to speak by her boy's deliberate choice of communication format - not face-to-face, not via speech, but the choice of a social networking service. Thus the shape of the modern family: to be sure, in theory always there, ready for communication; but in practice an entity where acts of communication need special care. 


\subsection{The homeless and family communication}

All the communications referred to in these thumbnails were interchanges between parents and their offspring. They are, also, and of course, distributed communication that is, these interlocutors are not being described as in unmediated face-toface contact but are conducted in a situation where the parent(s) and offspring are speaking from different spatial locations; they are remote from each other. It is a truism to say that, in the past, spatial remoteness was more likely to engender a lack of communication, while today technologies have so increased the possibilities of communication that distance is no longer the prohibitor it once was. Even when using the mobile 'phone, however, distance can be a factor that is oriented- to by the interlocutors in their communications with each other. But what these sketches are showing is that the technologies of communication have become a different sort of resource - they are not just 'solving' problems of distance. The various experiences afforded by different modes of communication - with Skype offering immediate visual and auditory availability, for example, while email a degree of asynchronicity, Facebook postings noticings by more than one other - all carry with them various orders of interactional consequence. There are benefits to seeing and being seen; there are benefits to 'posting' and being read (Harper, 2011). Grossly speaking, what is synchronous leads to certain sorts of interactional consequences; what is asynchronous others; what is private and 'one to one' different from 'one to many' - even if, as we saw, the latter is used for publicly showing 'one to one' communications.

Choice between these affordances, if you like, is made through consideration of the interactional management of the communications at hand. In particular, and in ways we have only just begun to detail, such considerations may include how to re-balance or shift control between the interlocutors. As we shall see later, this potential may be particularly relevant to the asymmetries of control located in social conventions of 'parent-offspring' interaction; conventions and systems of propriety that very often apply in the face-to-face situation too, but which have an especial salience when one of these parties have left home. For the status of homeless young person seems to imply something about the order of communication that existed before that status is acquired.

To explore this point: one might suggest that parent/offspring relations are ordinarily governed by a rule that holds that, whatever their age (mutatis mutandis), offspring are "answerable" to their parents; they should be accountable to them, respond to their directives, etc. (see for example Aronsson \& Cekaite 2011). That this is so might be brought into the foreground of communications when one of these parties has chosen to be physically separated from the other. In other words, being homeless implies something about the rules of family communication.

The notion of rule here is important and we shall say more about this later. But as we shall see, rule is being used to label an orientation that, in ordinary 
circumstances, the parties involved take heed of and demonstrate that they do so in their interactional choices. The issue here is that the situation might not be 'ordinary'. Abiding by a maxim that says one is answerable to one's parents has a different inflection when one has left the home which one shared with the parents; this family is no longer 'ordinary'. One of the ways that this 'unordinary fact' manifests itself is in how these technology-mediated situations we are here describing constitute locations in social life where the participants are, in a sense, behaving with peculiar thoughtfulness: they do so in ways that are 'strategically-orientated' with regard to this fact that their situation is peculiar. They make more or less explicit choices as between modes that best suit their interests and purposes - this is not casual, thoughtless chit-chat.

Following Garfinkel (1967, 96-103) (who used the term in a different context), we might consider this a 'common sense situation of choice' which is a special situation, even if the choice-making 'cultural machinery' brought to bear has its more general roots in a broader range of everyday practices. Here more generally-used devices of communication is specifically designed to incorporate a situation where one of the parties is avowedly 'without home'. Such reciprocal 'recipient design techniques' delivers part of the actual meaning conveyed in the communication in that context. To communicate in a manner where the recipient cannot respond visually, there and then, for example, is treated as or is assumed to be cast in terms of an 'in-order-to chosen' cultural motive - in order, for instance, to hide facial expressions. To communicate face-to-face or through the digital equivalent (via Skype say) has a similarly 'strategic' in-order-to cast, though with obviously different consequences - here 'showing' and 'being seen' is evidently what is sought for and again this might have something to do with what can (or is desired to be) be seen when one party is conceived as homeless and remote. To communicate is not, then, just a question of being in touch; it's about the publicly motivated status of communicative acts that adapt them to the specific known-in-common constellation of social relations, the relations of family-life-in-this-case. Here, though, those social relations have a peculiar premise: that one member of a family has become avowedly homeless; not only they but the family as a whole thus acquire a very specific moral profile.

\subsection{Identity, communication, participation frameworks}

Such considerations are derived from the thumbnails we have offered so far, but we trust that readers will bear them in mind as we move on to the rather more complex and extended case studies we now offer, where these considerations are frequently found in complex combinations. Let us now turn to Sample 2 for a somewhat more extended illustration: 


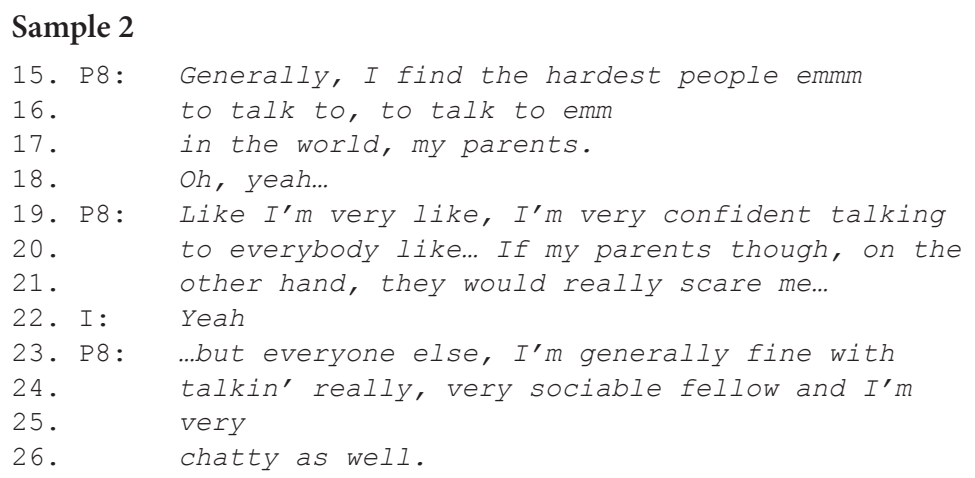

We might say that this Sample shows the 'identity-rich' nature of communication or how the specific communication is shaped by the identities of the interlocutors. The identities 'parent' and 'fellow' serve as organizing items in this transcript along with self-descriptions of the respondent as "chatty", "sociable". These identities can be conceived of as 'membership categorizations', i.e. as one of a vast range of commonsense equivalence classes which serve as loci for the imputation of rights and obligation and other predicates (originally Sacks 1972a, 1972b; for current thinking on the concept, see Watson in Fitzgerald \& Housley 2015). This respondent cast his self-description of communicative performance in terms of the membership categorization 'parents'. The selection of this categorization along with the possessive pronoun 'my' (parents) projects a counterpart category, 'offspring', and thus creates a category pair, 'parent-offspring'. Sack's terms this creation a 'relational pair'. The description of our respondent's communicative performance or fluency is cast in terms of this kind of pair. He describes his parents as 'the hardest people to talk to in the world', where they would 'really scare' him. By contrast, when the category 'everybody', 'everyone (else)' is invoked as the contrastive communicative locus, then the respondent self-describes as 'very confident', 'chatty' and 'sociable'.

One might consider that his inhibitions as regards speaking with his parents is thus a generic thing, not just something that occurs in discourse mediated by communication technologies. The inference is available that there is a 'natural history' to that avowed inhibition. But there is another additional inference that is possible here: that his categorization - based account also furnishes grounds for the selection of a particular technological mode for communication with his parents, e.g. an asynchronous mode such as texting, a mode which, unlike Skype, say, does not involve mutual visual availability when the history of their relationship suggests this might be desirable.

A person's orientation to some membership categories may also include the knowledge that some are 'territorially-based', to use Schegloff's term (1972; also 2005). This is clearly relevant here since the 'territory' of the categorization 'parent(s)' 
is known to be the 'family home', a special area that is, as it were, 'owned' by the parents (Speier 1971). This is a case of a more general feature of 'society-as-it-is-commonly-known', which Schegloff expresses (for other purposes) as follows:

Although the structure of knowledge about a 'sort of place' may be general and formal... the particulars that are so organized are assumed to vary with territorially-based memberships. Thus, most persons live similarly, in a place in an environment of places, in a house, a neighbourhood... which can similarly be talked of (and it is an important fact that some do not). These categories are filled by persons with their particular situations, their house, their street.

$(1972,101)$

The 'parents' in the 'parent-offspring' relational pair have different claimable rights and obligations than the 'offspring' in terms of location and territory, then. The place known as "the house" "belongs" to all the family ("my house", "my home"), though a fortiori to the parents - hence the term 'parental home'. As a consequence of this, parents may be conceived as being able to invoke 'special rights' over that place: for instance, they may warn their offspring "while you live under our roof you must keep to our rules", for example (note the use of the possessive pronoun 'our'). This is but one of the many category-formed asymmetries engendered by the 'parent-offspring' family type and, of course, one inference that may be drawn, at least as an extreme case, "if you constantly break our rules you must leave".

Pursuing this point: certain conversational topics may be nominated by parents as 'sensitive'. These might include topics concerning drugs, sex (and sexuality), music, friends and associates, even timekeeping. We are not suggesting that these are the topics that parents elect to be sensitive, but suggesting that parents have, or can claim, the right to make such elections. Once defined, such topics may be largely avoided in face-to-face situations by offspring who may wish to abide by their parent's 'rules' about such topics. On the other hand, offspring might use technology to 'work around' parental impositions. Indeed the constraints on parent-offspring communication so often seen as a 'problem' to be 'overcome' when in the family home, may in fact come to be a resource for reshaping the topics of 'talk with mum and dad' when technology is used to enable spatially distributed communication. For one thing, the offspring might claim that they are out of the space in which those constraints apply.

Of course the reverse might hold true: it might allow the parents to raise topics that the child might want to resist. Consider the following sample.

\section{Sample 3}

93. I:

94.

95. P8:

96.

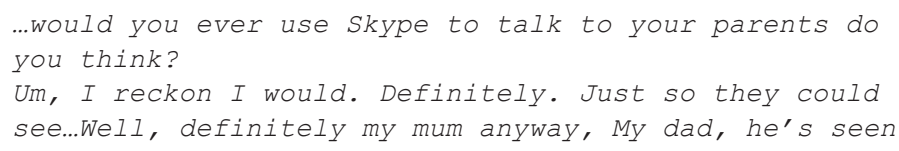




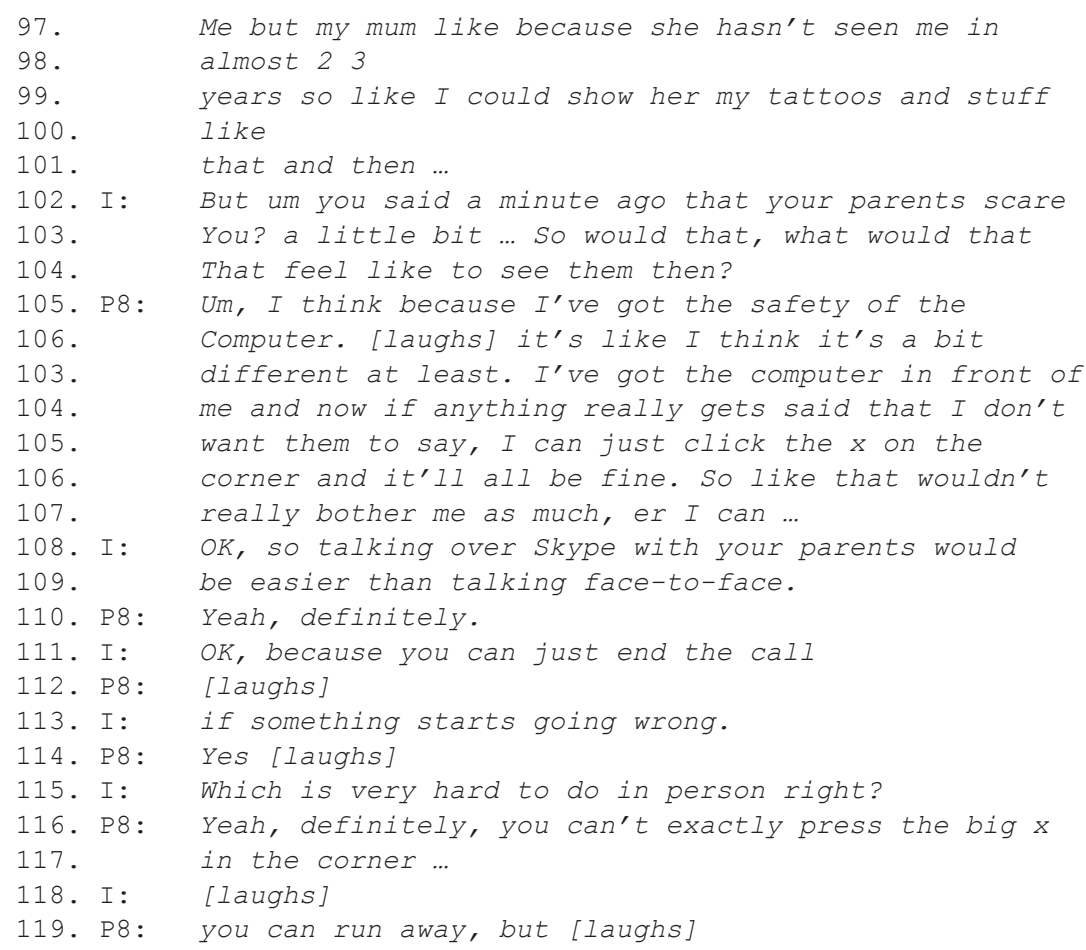

Here, the lines 105-119 indicate the perceived utility to the homeless young person of distributed positions (mediated by a computer) as between parents and offspring. He says that in unmediated face-to-face interaction there is no 'big $\mathrm{x}$ ' in the corner whereby the interaction could be 'switched' off'. Computers afford just that facility, if, for instance, something "starts going wrong" (line 113) or when (line 104-5) "something gets said that I don't want them to say", (e.g. sensitive topics, again). Such affordances are both glossed and specified by the respondent as "... the safety of the computer" (line 105-6). Thus the very means that permit distributed communication also permit, purpose-designed, situationally-sensitive, often innovative or improvisational modes of control over that communication - and this is a two way street': just as it allows the homeless young to control that communication, so it can allow others to do the same, -parents, in this case.

The distributed and mediated nature of the contract may be used as resources in several ways, not least to do with sensitive topics. It might also allow the communicative management of emotional states or 'states of mind'. Note that the respondent in Sample 2 claims that face-to-face talks with his parents "really scare (him)". The respondent in Sample 4, meanwhile, regards texting as managing his mother's "mood", particularly when she was and remained, angry with him. 


\section{Sample 4}

\section{I:}

121.

122. P6:

123. I:

124. P6:

125. I:

126. P6:

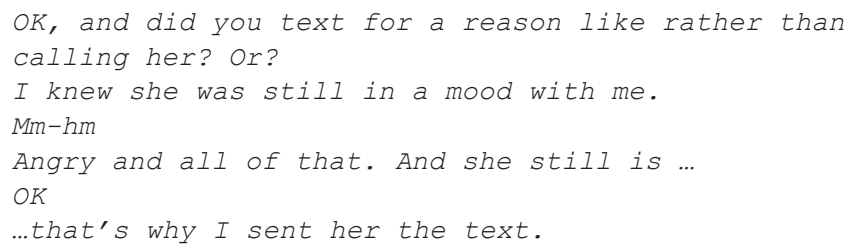

In Sample 5, however, we find further considerations, particularities that are mentioned to explain the particular situation the respondent wants understood. As we see, he describes his mother as 'bipolar' and one who could "just switch" emotionally (lines 43-44). The use of the 'phone affords the protection of spatial distribution but being synchronous can establish a 'state of talk' which, as such, doesn't entirely occlude the possibility of an emotional switch but does provide for the possibility of 'hanging up' and 'ringing back' (line 56). Note too that texting plays its practical role providing for these communications, the asynchronous nature of this mode permitting the leaving of a message rather than entering into a reciprocal 'state of talk'.

\section{Sample 5}

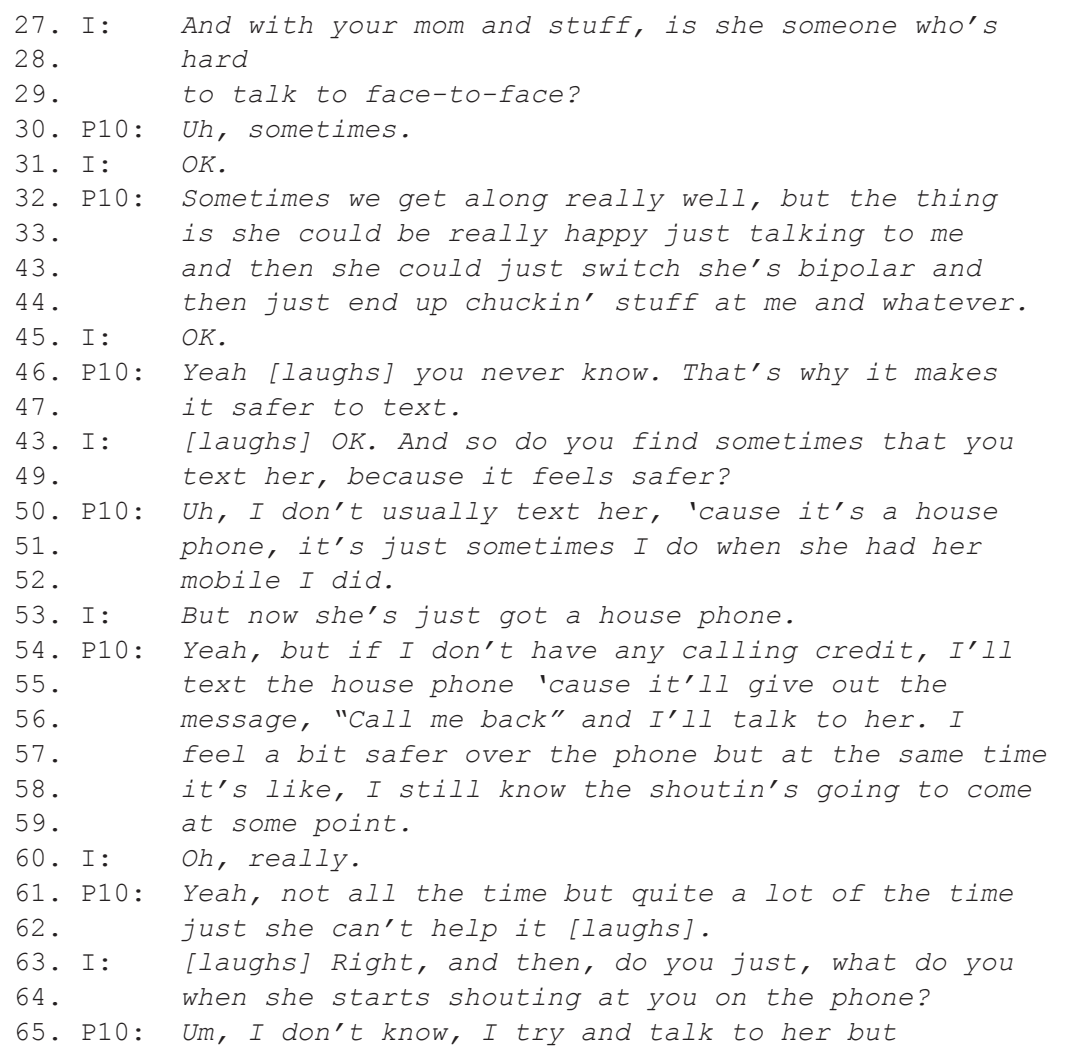




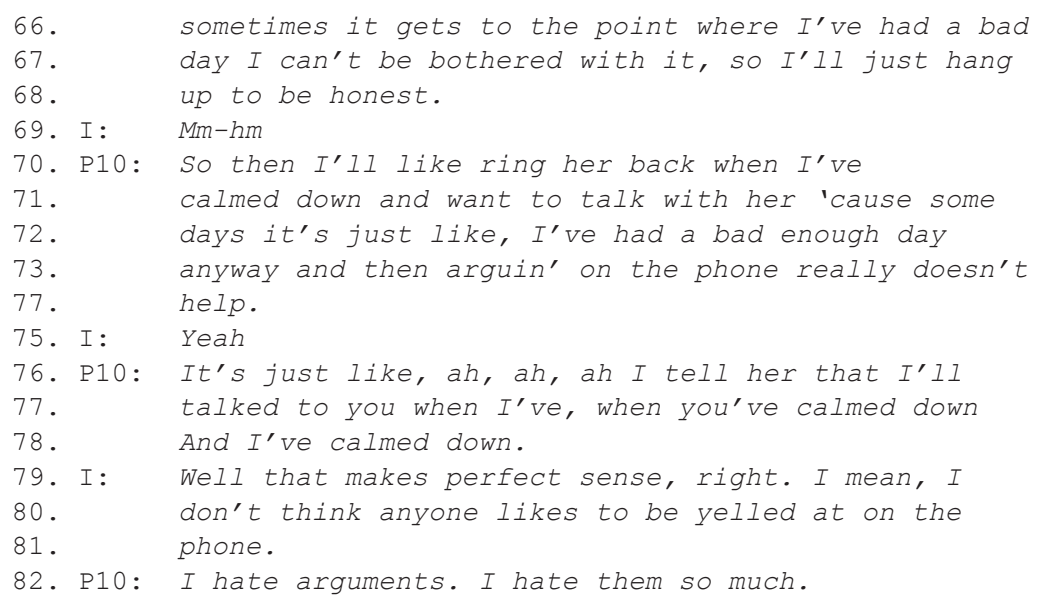

Reflexively, the choice of a particular technology allows the homeless young to manage their own conduct. Note that the respondent in Sample 6, below, usually has disagreements with her mother over the 'phone. She prefers to use this technology because with it, he can 'hang-up', (line 143) and thus close the conversation off. Key to this is, of course, the distributed nature of the mediated interaction, a spatial arrangement which means that the subject can 'stay away' from her mother for a while. Indeed, she can choose to remain 'remote', and thus out of touch until she is minded to alter the situation - perhaps turning to the phone again.

An additional issue is that the visible features of the disagreement/argument are not available on the 'phone and so by choosing this technology the respondent can avoid seeing - and dealing with - her mother's upset. For instance, visible upset can intensify an oral-aural disagreement, and thus the use of a non-visual means can serve as a means of diminishing rather than intensifying a disagreement or disaccord. In a way, this can move the communication toward the normative preference for agreement (Sacks 1970), by at least occluding the intensification or escalation of a disagreement.

\section{Sample 6}

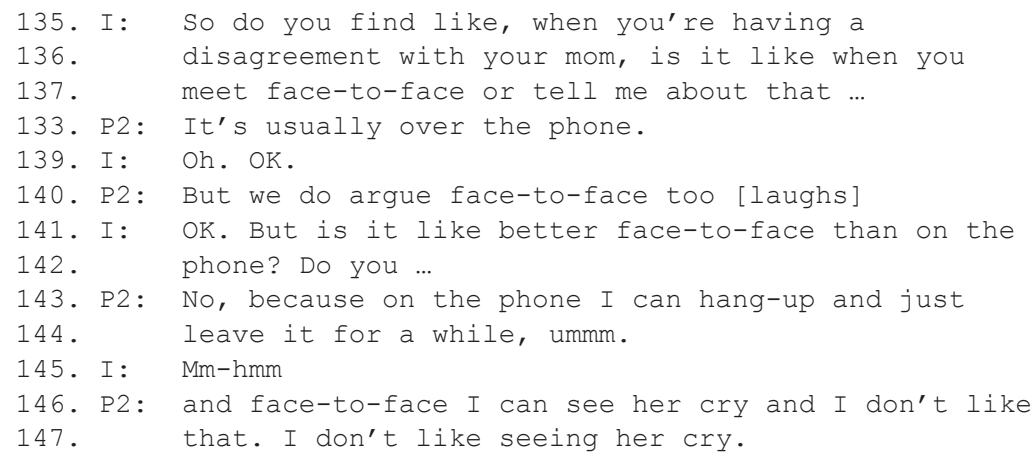


To sum up: the choice of particular modes such as 'phoning, texting, video telephony via Skype or the use of social media like Facebook can provide for the management of particular kinds of emotional expression. By 'management', we also intend the occlusion or forestalling of, say, the onset of emotional outbursts or upsets as well as the management of their course post-onset. One important dimension we are highlighting is the synchronicity of a mode, but this is mediated also by the scale or form of a mode: 'talk-only' calls on mobile phones, perceivedly permit of closing down the 'state of talk' without the complex interactional disengagements that might be entailed with calls that are also visual, such as those conducted via Skype. As the samples show, conciliations and compromises that face-to-face closings might require can be irksome to the homeless. This is not because the management of closings is difficult in ordinary communications, but because of the character of those involved in homeless young person communications to their parents can make them so. To say goodbye might require a joint agreement in typical conversations, but this is much more difficult when one party shows visible dimensions of, say, emotional distress. Such a state can preclude the warrantable introduction of 'pre-closing utterances' (Schegloff and Sacks 1973). Then, seeking closure is a much more complex and open-ended, given that a perceived 'emotional state' can typically occasion a disclosure rather than a closing sequence. For interlocutors, the perceptible existence of such emotional states in the communication can be laden with implications about the unfolding of a relationship; to be homeless here may point to that state as a comment on a relationship - the one embodied in the communication about to come to an end. No wonder then that modes of communication that can affect endings matter.

The samples all show then that the self-avowed homeless young have to deal with subtle and complex management issues in their communications - more so than might apply for 'normal' family communications. Distance, being spatially separate from the other, is an important resource, the above shows; it is also the source of trouble. When using Skype, for example, the potential interaction this affords, allowing reference to the visual and so on which might well be worrisome to one or both parts ('see her crying' etc.), nevertheless allows radical steps of closure that are not available face-to-face - there is always the "big $\mathrm{x}$ in the corner (of the screen)", as P8 puts it in Sample 3. Whatever the seeming abruptness of such a disengagement, it is perceivably preferable to the alternatives - suffering the escalation or aggravation, perhaps having to have to go through complex, difficult procedures of compromise or "backing down", losing face in relation to a position to which one is committed and so on. We might term this perceived affordance of Skype - or rather, this Skype-related practice - a 'selective intimacy', one that affords more radical management for the parties than normal face-to-face action. This is clearly bound up not with Skype alone, but with how this medium (so to speak) gets used in particular relations: between the homeless young and their 
parents. The outcome of all this is that the choice of different digital platforms can be highly sensitive to the specific 'footing' of the particular relationship, as well as being able to play a part in the active reproduction of that self same footing. ${ }^{1}$ That is, interlocutors' practices (not just technology related practices, of course) establish a basis - what Goodwin later termed a 'participation framework' - upon which these interlocutors can premise their further practices; many such frameworks, which show minute 'recipient design' and other particularising features, manifest the kind of asymmetries to which we have tried to point in this paper. The use of different technologies can greatly enhance a given participation framework.

What is clear from this evidence, also, is that for these homeless young people, 'homelessness' does not necessarily connote being ex communicado. But what we also see is a particular preference organisation in the communication that they do have, especially with their parents. This is where the artful selection and use of a given mode comes in, whether to text or Skype, to email or post on Facebook. Such selection shows that another of the connotations often conventionally associated with the identity 'homeless person', namely a deficit in competence, is not relevant here. Our respondents show skills of control even over the fine detail of their interactions with others and particularly over the type of availability and presence they will accept or permit with parents. Choosing when to be in touch and when to be out of touch is key to this.

Controlling communication is not simply about who communicates, then, but the order of communication. This order is massively to do with sequences; with what our respondents explain as what leads on from another. To out this analytically, this has to do with 'turn taking systems'. A major feature in the sequential or serial organization of conversational actions in the communications we have been describing has to do with 'paired actions', and, in particular, the production of a 'conditionally-relevant' (Schegloff 1969) second action by a second party consequent upon the production of a first action by the initiating party. One property of these adjacency-paired actions is that in face-to-face conversational interaction they are the only conversational device that can 'make something happen next, as an immediate next action'. However, whilst next actions remain oriented-to by our respondents, the issuing of a next action-say, an answer to a question or a response to a criticism, is more complicated - it becomes bound to the medium of communication chosen. As we have seen, this is at once a resource and constraint for decision making since it allows a different order of pairing and hence next turn acts.

If, for instance, the first of the paired action sequence is texted, then a second action remains conditionally relevant, but may not be immediately produced.

1. On 'footing', see Goffman 1979, and Goodwin's move towards an ethnomethodological re-specification of Goffman's conception, in e.g., Goodwin 1981. 
Instead, its production may be delayed for a day or two or more: this is a matter that is accounted for as routine in this specific form of technology-mediated communication, in the communications between the homeless young and their parents. Consequently, what might otherwise be seen as a 'hiatus' in the production of a second action to the pair is deemed understandable by virtue of the technology medium being used: the requirement of immediacy of its production may be relaxed. This is one way in which a particular medium can be used as an interactional resource by, say, the parent or - and this seems more likely given the data samples presented thus far - the offspring. Of course, the selection of a second action as an immediate matter is much closer to the face-to-face requirements in other media, e.g., Skype or a voice only communication.

The frequent relevance of 'next action' considerations in the interactions between the homeless young persons and their parent(s) is, we want to note, often attested to in our data. For instance, in Sample 7, respondent P2 attests to the relevance of her mother not responding to her calls or messages. The non-production of a second pair-part action from P2's mother when P2 has issued a first pair-part action is clearly seen as a 'complainable':

\section{Sample 7}

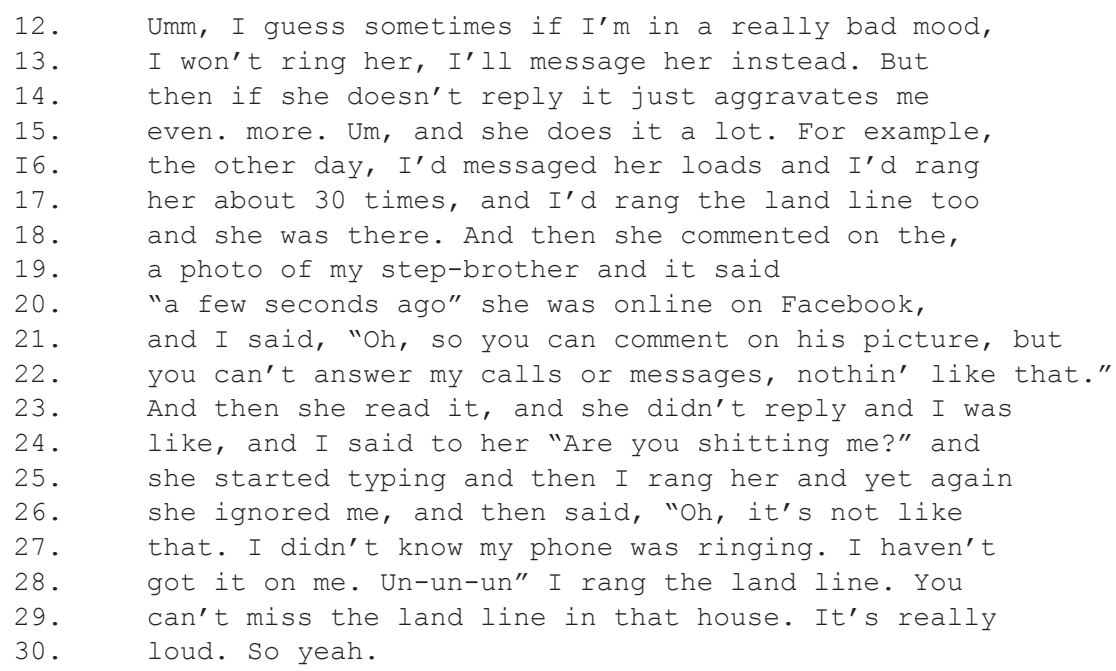

Here we can see that the non-production of a second pair-part conversational action can occasion a complaint. As Schegloff (2005) analyses (developing ideas from Sacks 1987, Sacks et al. 1974, and Sacks \& Schegloff 1979), the non-production of a second action is, for interlocutors, inferentially-rich and morally-accountable. Here, the respondent aggravates the complaint that her mother "can't answer (her) calls and messages, nothin' like that" (line 22) by creating in the interview a 'contrast 
case' concerning the mother's response to another first action of a pair, her brother's showing of a picture. Through this initial case in the contrast, a rule is highlighted "respond to first pair part actions" - which is then shown to be breached in the case of the mother not replying to her calls and messages. Observe, also, that the mother's attempted excuse is dismissed as implausible by the interviewee, on the grounds that the landline phone, allegedly, rings loudly (line 30). Thus, a delay may be allowable in, say, the text medium but as this excerpt suggests, there is a 'statute of limitations' in the amount of time in which conditional relevance pertains. This is, perhaps, particularly the case when there is an 'externally-imposed' time limit for the reply, e.g., when an appointment becomes due. Consequently, there are delays and delays, depending, for instance, upon the particular circumstance of each call. Here, a first pair-part action, an 'informing' does not (or, perhaps, not in time) receive a second pair-part expression of receipt, and it is this that creates a complainable from the daughter. As Schegloff and Sacks (1973) observe, in regular circumstances, the non-provision of a second pair-part, say, a response, by a recipient of a first pair-part - say, an announcement - is conventionally treated by the announcer as a noticeable matter, as the recipient's responsibility, and as inferentally-rich, i.e., the announcer can draw inferences about the recipient on the basis of the non-response. Such a reaction may well apply, mutatis mutandis, to circumstances such as those depicted in this illustration.

\subsection{The 'politics of living' in the detailed organisation of communications}

We are explicating the social organisational phenomenon of homelessness through an investigation of the patterned ways that the term 'homelessness' and those categories of person attendant on it - family, mum, dad, son, daughter, child, and so on - are deployed as part of the interactional order of interviews. In this focus, accounts offered by those interviewed have not being treated as freestanding evidence of homelessness and its causes, but rather as part of a tool set of practical resources through which both the interviewed persons and those doing the interviewing - us - jointly infer and elaborate a world known in common, as the one that is at once treated as the real world and about which sensible detailed things can be said; these details have implications for what we earlier called the 'politics of living' - implied next acts, correctives, routines, histories. We feel that this term applies equally aptly to Jackson's (2012) observations concerning the parameters of mobility of homeless people in the city.

In particular, we have noted how the homeless young describe their various technology-based communicative choices as intended to achieve specific forms of enablement and control over their dealings with parents: this is the 'politics' of their 
conduct. Their choice-making activities constitute attempts to re-balance their relations, shifting the footing of one party vis-à-vis the other (Goffman 1981), which here, following but adapting Goodwin (1981), we have re-specified below in terms of participation status and participation framework. Very frequently, the choices accounted to us afford the offspring a margin of autonomy and standing that they might not have had in the home, or face-to-face situations.

In attempting to move toward a more analytic understanding of (in the present study) of these 'calibrating practices' we are pointing to some salient elements of those practices whilst also noting that their meaning, their functional significance, is related to a broader pattern which, following Garfinkel's interpretation of Gurwitsch's notion, one might call a 'gestalt contexture'. Such contextures are 'autochthonous'; that is to say naturally occurring, organised and situated. As Liberman $(2013,43)$ puts it, these are phenomenal fields, patterned arrays of details where the salience of the arrangement and hence their pertinence is achieved though participants' methodical work; this is how the dwelling spaces of existence come to have the form they do (Ingold 2102). These dwelling places are characterized in large part by the parties involved and their 'participation framework' (Goodwin 1981).

As we have noted, our homeless young methodically brought to bear, in interviews with us, their relationships with parents as constitutive of their strategic and practical orientation to being homeless. Their articulations about how to manage communication were central to this. These frameworks should not be thought of as constituting a structure through which people act, or which frame what they can and cannot do; it is rather that the framing is expressed in the work that our subjects undertake. This may be contrasted with the original formulation of the concept 'participation framework', where (e.g., in Duranti and Goodwin 1992) these frameworks are viewed as something resembling a structure that "align[s] speakers to hearers and actualize a state of discourse". However, our illustrations suggest that our respondents produce and ongoingly renew the participation framework. The weave of their interactions with their relations thereby take the form of achieved features of their interactions: the daughter's complaint about her mother in Sample 7, like the other examples we present, are the mechanisms whereby the practices of those involved come to be seen as properly a parent's and a child's. The homeless young in our data establish both their (commonly-known, activelyreproduced) 'participation status' and the interwoven activities that we refer to as the 'participation framework' (with parents) within which that 'status' operates in and through their talk-in-interaction, not as a consequence of a putative structure 'within which' their speech occurs. We may also note that the asymmetries that are produced and reproduced through these activities are not necessarily treated by interlocutors as negative things: sometimes these asymmetries are known, oriented-to, deliberately sought, intendedly achieved, and actively exploited. 
It is not, of course, implied that the management of participation frameworks is confined to the homeless young and their parents. What is especially notable is how the general resources 'going into' these frameworks (that is, the way they constitute systems of moral implicativeness associated with the categorisation of members and members' doings), gets particularised, fitted to the distinctive circumstances of each and every young person and their respective families. It is the particularised, intersubjective histories that give the participation frameworks invoked with regard to family relations the life and specificity they achieve in speech, in the interaction between subjects and interviewer, in this case, between us and the homeless young represented in our data and our illustrations.

The choice-making strategies we describe focus heavily on technologies of communication. Choices on this matter are not, of course, only a concern to the homeless young or their parents. Indeed, the motivations for, say, texting rather than talking on a mobile may be quite general. Other studies have shown how this is commonplace. Horst and Miller (2006), for example, note how low-income Jamaicans will employ texting rather than talking in order to avoid 'emotional " situations. More recently, Miller \& Sininan (2014) have explored how Skype is a managed and controlled choice between alternatives, used in particular ways to sustain remote family relations in diasporic communities. However, these studies tend to eschew a concern for the particularities of utterances about these choice strategies, tending to gloss them in stylised ethnographic reportage.

For our study, and by way of contrast, samples of interview talk show how 'general strategies' get invoked by the young people to characterise their own world-specific purposes; as a starting premise for conveying their circumstances, their problems in the interview context. We are arguing that there is good reason to examine homelessness in and through such particularising data. This follows not just the early groundwork of Anderson, but echoes too the turn to the linguistic powerfully articulated by Maynard (1988). Our data show that being homeless is a particular tool in the work of 'complainables' in talk (Schegloff 1995, 2005). It shows as well that the ways in which formulations about the 'features of homelessness as a social state' are elaborated points towards how difficulties-in-talk between parents and their children can be one of the more salient 'causes' of being homeless prefigured in accounts about current activities - as regards choices of communications media, for example. This should not be taken to mean that the possibility that it is 'troubles talking with parents' should be compared with other 'measures of truth' if these could be found. Rather, this constructed set of causal historical implicatures for an agreed current state (homelessness) are part of the resources through which the facts of homelessness are to be seen and understood. Whatever one might construe as the 'real facts', it is through the autochthonous work of formulating homelessness and its causes as implied in talk that understanding homelessness as a naturally occurring, ordinary phenomenon comes to be made visible. 


\section{Conclusion: Reasons, causes, evidentiality}

Such formulation work is not only about past events, of course, but is a route through which the 'homeless young' furnish their identity and account for their activities in the present. How our recipients tell where they are now implies, or often makes somewhat explicit, a route to that current point, even if the route is not always well specified. We have seen, in particular, how interactions between the young and their parents are formulated. These formulations assume that such relations have 'natural, everyday problems', accountably related in large part to the asymmetry of obligations constitutive of parent-child relations.

However, the formulations, a handful of which we have begun to analyse here, imply that these 'natural facts' turn out to have or become the source of chronic, even catastrophic difficulties for these young people and their parents. The interactions accounted for in our interviews might seem to entail 'talking with my mum'-type scenarios but on examination, they are not (always) the 'typical talk with my mum'- type events.

Though, at first glance, difficulties talking with one's parents seems an unlikely reason to become homeless, we have seen in a few pieces of evidence how homeless young people, in interview and interaction with 'experts' (us), articulate this as a 'reasonable-reason-to-leave-home' or at least, a 'reasonable reason for me to be in this situation' viz, 'homeless'. In this respect, these accounts turn on the presumption that the world and its organisation is known in common both by the homeless and those interviewing them. Although not everyone might make the decision to avoid conversation by making the radical decision to leave home, that those reported here chose to do so can be seen to be a 'fact of life' that one - that anyone - can understand as a rational 'cause' of action; hence our allusion to Garfinkel and his notion of choice.

The kind of illustrative material we have put forward might suggest that the exact status of the claim that 'difficulties in talk with my mum' needs to be carefully presented. At first consideration, one might be lead to think that 'proof' of this claim would be found not in the kinds of data we have - interviews, design sessions and such like - but in (or through) transcript data of 'real' conversation between parents and their offspring; data of in vivo interaction. However, and as we have seen, a crucial property of homelessness as a social category is that it is deployed in contexts where it is a given - or rather in our interview contexts it is a given. Though an ideal world from a researcher's point might allow data to be captured prior to the status of homelessness being a fact, a desire for this would be akin, let us say, to a criminologist wanting to be present at the moment someone undertakes a crime - rather than only through the post hoc understanding of that crime when the criminal has been caught and charged. 
Part of the desire here, the desire for better data, is bound up with a feature of the categories in question - both with regards to crime and homelessness. For part of the categorisation that unfolds once someone has been charged and convicted of a crime is the retrospective re-characterisation of that person as being a criminal and always thus; as being a person who could have been predicted to behave so because they were (somehow) always bad by inclination. Similarly with homelessness and the homeless: once categorised as such, the prior history of a homeless person is, to some degree, recast as providing the reasons, the 'causes' for their current predicament. Of course other social problem categories may have different properties that don't evoke a reconstituted causally patterned past in just this way - as ones 'who were bound to become homeless'.

Nevertheless, an important point about this is that though the past is evidently important in nearly all social affairs and without wanting to deny the merits of in vivo data of the kind alluded to, the desire for 'events in the past' can lead the eye away from consideration of the current - present time - circumstances in which this definitional work about the past is being done. The past that leads to homelessness is, in a sense, constructed through the sometimes 'prismatic' powers of language use in the present, in the here and now. Some of the properties of this prismatic work can be lost given the peculiar property of the category in question. We are noting that how this works tempts people to imagine how good it would be if they could have been there - in the past when the cause or event that led to homelessness occurred; it leads the eyes and the heart away from the here and now, the office in which some interview is being done in to a grief laden past of hurt and unintentional cruelty, a world in which ordinary kids leave home for all too understandable reasons only thereby to make themselves far from ordinary - kids without 'normal' mums and dad, kids without the normal given - that they have a home to go to, that they have a mum and dad that they can turn to and Skype to say, 'Can I come home?'

\section{References}

Anderson, N. 1923. The Hobo: The Sociology of the Homeless Man. Chicago: Chicago Council of Social Agencies.

Aronsson, K., and A. Cekaite. 2011. "Activity Contracts and Directives in Everyday Family Politics.” Discourse and Society 22 (2): 137-54. doi:10.1177/0957926510392124

Bourgois, P. 1998a. "Just Another Night in a Shooting Gallery." Theory, Culture and Society 15 (2): 37-66. doi:10.1177/026327698015002002

Bourgois, P. 1998b. "The Moral Economies of Homeless Heroin Addicts: Confronting Ethnography, H.I.V. Risk and Everyday Violence in San Francisco Shooting Encampments." Substance Use and Misuse 33 (11): 2323-51. doi:10.3109/10826089809056260 
Castells, M. 1996. The Rise of the Networked Society: The Information Age: Economy, Society and Culture Vol. I. Cambridge, MA; Oxford, UK: Blackwell.

Castells, M. 1997. The Power of Identity, The Information Age: Economy, Society and Culture Vol. II. Cambridge, MA/Oxford, UK: Blackwell.

Castells, M. 1998. End of Millennium, The Information Age: Economy, Society and Culture Vol. III. Cambridge, MA/Oxford, UK: Blackwell.

Duranti, A., and C. Goodwin. 1992. "Editors' Introduction", Rethinking Context, ed. by A. Duranti, and C. Goodwin. Cambridge: Cambridge University Press.

Fitzgerald, R., and W. Housley. 2015. Advances in Membership Categorisation Analysis. London: Sage. doi: 10.4135/9781473917873

Garfinkel, H. 1967. Studies in Ethnomethodology. Englewood Cliffs, New Jersey: Prentice Hall.

Garfinkel, H., G. Girton, E. Livingston, and H. Sacks. No date. Studies of Kids' Culture and Kids' Talk. Manuscript.

Garfinkel, H., and H. Sacks. 1970. "On Formal Structures of Practical Actions." In Theoretical Sociology: Perspectives and Developments, ed. by J. C. McKinney, and E. A. Tiryakian, 337366. New York: Appleton-Century Crofts.

Goffman, E. 1979. “Footing." Semiotica 25 (1-2): 1-29. doi:10.1515/semi.1979.25.1-2.1

Goodwin, C. 1981. Conversational Organization: Interaction Between Speakers and Hearers. New York: Academic Press.

Harper, R. 2011. Texture: Human Expression in the Age of Communication Overload. Cambridge, Mass.: MIT Press.

Horst, H. A., and D. Miller. 2006. The Cell Phone: An Anthropology of Communication. Oxford/ New York: Berg.

Ingold, T. 2011. Being Alive: Essays on Movement, Knowledge and Description. Abingdon: Routledge.

Jackson, E. 2012. "Fixed in Mobility-Young Homeless People and the City." International Journal of Urban and Regional Research 36 (4): 725-41. doi:10.1111/j.1468-2427.2012.01124.x

Katz, J., and M. Aakhus. 2002. Perpetual Contact: Mobile communication, Private Talk, Public Performance. New York: Cambridge University Press. doi:10.1017/CBO9780511489471

Latour, B. 2013. An Inquiry into Modes of Existence. (Trans C. Porter). Harvard: Harvard University Press.

Liberman, K. 2013. More Studies in Ethnomethodology. Albany: State University of New York Press.

Maynard, D. W. 1988. "Language, Interaction, and Social Problems." Social Problems 35: 311-334. doi: $10.2307 / 800590$

Miller, D., and J. Sinina. 2014. Webcam. Cambridge: Polity Press.

Rose, E. 1960. "The English Record of a Natural Sociology." American Sociological Review 25: 193-208. doi: $10.2307 / 2092625$

Sacks, H. 1972a. "On the Usability of Conversational Data for Doing Sociology." In Studies in Social Interaction, ed. by D. Sudnow. New York: The Free Press.

Sacks, H. 1972b. "On the Analyzability of Stories by Children." In Directions in Sociolinguistics: The Ethnography of Communication, ed. by John J. Gumperz, and Dell Hymes. New York: Holt, Rinehart.

Sacks, H. 1987. "On the Preferences for Agreement and Contiguity in Sequences in Conversation." In Talk and Social Organisation, ed. by G. R. Button, and J. R. E. Lee, 54-69. Clevedon: Multilingual Matters.

Sacks, H. 1992. Lectures on Conversation. Volumes I \& II. Malden: Blackwell. 
Sacks, H., E. Schegloff, and G. Jefferson. 1974. "A Simplest Systematics for the Organization of Turn-Taking for Conversation.” Language 50 (4): 696-735. doi:10.1353/lan.1974.0010

Sacks, H., and E. Schegloff. 1979. “Two Preferences in the Organization of Reference to Persons in Conversation and Their Interaction." In Everyday Language: Studies in Ethnomethodology, ed. by G. Psathas, 15-21. New York: Irvington Press.

Schegloff, E. 1969. "Sequencing in Conversational Openings." American Anthropologist 70: 1075-95. doi:10.1525/aa.1968.70.6.02a00030

Schegloff, E. 1972. "Notes on a Conversational Practice: Formulating Place." In Studies in social interaction, ed. by D. Sudnow, 75-119. New York: Free Press.

Schegloff, E. 1995. "Discourse as an Interactional Achievement III: The Omni-relevance of Action." Research on Language and Social Interaction 28 (3): 185-213. doi:10.1207/s15327973rlsi2803_2

Schegloff, E. 2005. “On Complainability.” Social Problems 52: 449-476. doi:10.1525/sp.2005.52.4.449 Schegloff, E. A., and H. Sacks. 1973. “Opening up Closings.” Semiotica 8: 280-327. doi: 10.1515/semi.1973.8.4.289

Speier, M. 1971. "The Everyday World of the Child." In Understanding everyday life, ed. by J. Douglas, 188-217. London: Routledge and Kegan Paul.

Spradley, J. P. 1970. You Owe Yourself a Drunk: An Ethnography of Urban Nomads. Boston: Little, Brown.

Watson, R. 2015. "De-Reifying Categories." In Advances in membership categorization analysis, ed. by R. Fitzgerald, and W. Housely, 23-49. Los Angeles: Sage. doi:10.4135/9781473917873.n2

Woelfer, J., and D. Hendry. 2010. "Homeless Young People's Experiences with Information Systems: Life and Work in a Community Technology Center." Proceedings of the SIGCHI Conference on Human Factors in Computing Systems, CHI 2010, pp. 1291-1300.

Woelfer, J., and D. Hendry. 2011. "Homeless Young People and Living with Personal Digital Artifacts." Proceedings of the SIGCHI Conference on Human Factors in Computing Systems, CHI 2011, ACM Press, pp. 1697-1706.

Yoo, D., A. Huldtgren, J. Woelfer, F. Hendry, and B. Friedman. 2013. "A value Sensitive ActionReflection Model: Evolving a Co-Design Space with Stakeholder and Designer Prompts" Proceedings of the SIGCHI conference on human factors in computing systems, CHI 2013, ACM Press, pp. 419-428. doi:10.1145/2470654.2470715

\section{Authors' addresses}

Richard H. R. Harper

Social Shaping Research, Cambridge

England

richard@socialshapingresearch.com

Rod Watson

Telecom ParisTech, Paris

France

r.watson339@btinternet.com

Jill Palzkill Woelfer

University of Washington

United States

woelfj@uw.edu 